\title{
Concentrações plasmáticas de triptamina, tiramina e feniletilamina em eqüinos sob efeitos de sobrecarga de carboidratos e antiinflamatórios não esteroidais ${ }^{1}$
}

\begin{abstract}
Paulo Tarso L. Botteon ${ }^{\star}$, Vany P. Ferraz ${ }^{3}$, Maria Beatriz A. Glória ${ }^{4}$, Marília M. Melo $^{5}$, Fabíola O. Paes Leme ${ }^{5}$, Rafael R. Faleiros ${ }^{5}$ e Geraldo Eleno S. Alves ${ }^{5}$

ABSTRACT.- Botteon P.T.L., Ferraz V.P., Glória M.B.A., Melo M.M., Paes Leme F.O., Faleiros R.R. \& Alves G.E.S. 2008. [Plasmatic concentrations of tryptamine, tyramine end phenylethylamine in horses under the effect of carbohydrate overload and non-steroid antinflammatory compounds.] Concentrações plasmáticas de triptamina, tiramina e feniletilamina em eqüinos sob efeitos de sobrecarga de carboidratos e antiinflamatórios não esteroidais. Pesquisa Veterinária Brasileira 28(6):299-302. Departamento de Clínica e Cirurgia Veterinárias, Escola de Veterinária, Universidade Federal de Minas Gerais, Av. Antônio Carlos 6627, Pampulha, Belo Horizonte, MG, 30123-970, Brazil. E-mail: paulobotteon@gmail.com

The concentrations of the bioactives amines tryptamine (TRP), tyramine (TYR) and phenylethylamine (PEA) were determined by gas chromatography in plasma samples of 20 horses submitted to carbohydrate overload. Thirty hours after the overload, the horses were randomly distributed in four groups $(n=5)$ and were submitted to four IV treatments every 12 hours: $10 \mathrm{ml}$ of saline (GC), ketoprofen $2.2 \mathrm{mg} / \mathrm{kg}$ (GK), phenylbutazone $4.4 \mathrm{mg} / \mathrm{kg}$ (GF), and flunixin meglumine $1.1 \mathrm{mg} / \mathrm{kg}$ (GFM). Blood samples were collected at various times after the overload (0-72 h). Plasma TYR and PEA concentrations ranged from 0.18 to $164.2 \mathrm{mg} / \mathrm{L}$, and differed significantly with time $(p<0.01)$, but did not differ in the treatments. Plasma concentrations of TRP differed between times and treatments. The GC was significantly major than other treatments at $48 \mathrm{~h}$ and $60 \mathrm{~h}$ after the overload, and the plasma concentration of TRP in groups GK and GFM was significantly lower than in groups GF and $\mathrm{GC}$ at $72 \mathrm{~h}(\mathrm{p}=0.0012)$. We concluded that the anti-inflammatory drugs evaluated do not interfere in the plasma concentration of TYP and PEA. For TRP, ketoprofen and flunixin meglumine was effective to reduce de plasmatic concentration of this amine.
\end{abstract}

INDEX TERMS: Amines, antinflammatory compounds, laminitis, horse.

${ }^{1}$ Recebido em 31 de janeiro de 2008.

Aceito para publicação em 3 de março de 2008.

Parte do trabalho de Pós-Doutoramento do primeiro autor, apresentado ao Programa de Pós-Graduação em Ciência Animal, Escola de Veterinária (EV), Universidade Federal de Minas Gerais (UFMG).

${ }^{2}$ Departamento de Medicina e Cirurgia Veterinária, Instituto de Veterinária, Universidade Federal Rural do Rio de Janeiro, Seropédica, RJ 23851-970, Brasil. *Autor para correspondência: paulobotteon@gmail.com

${ }^{3}$ Departamento de Química, Instituto de Ciências Exatas, UFMG, Av. Antonio Carlos 6627, Pampulha, Belo Horizonte, MG 31210-901, Brasil.

${ }^{4}$ Departamento de Alimentos, Faculdade de Farmácia, UFMG, Belo Horizonte, MG.

${ }^{5}$ Departamento de Clínica e Cirurgia Veterinária, EV, UFMG, Belo Horizonte, MG..
RESUMO.- As concentrações plasmáticas das aminas triptamina (TRP), tyramina (TYR) e pheniletilamina (PEA) foram determinadas por cromatografia gasosa (CG) de 20 eqüinos sob efeito de sobrecarga por carboidratos (SC). Após $36 \mathrm{~h}$ da SC os animais foram aleatoriamente divididos em quatro grupos $(n=5)$ e receberam a cada $12 \mathrm{~h}$ por via iv: solução salina $10 \mathrm{~mL}(\mathrm{GC})$, ketoprofeno $2,2 \mathrm{mg} / \mathrm{kg}$ (GK), fenilbutazona $4,4 \mathrm{mg} / \mathrm{kg}$ (GF) e flunixin meglumine $1,1 \mathrm{mg} / \mathrm{kg}$ (GFM). As concentrações das aminas TYR e PEA variaram de 0,18 a $164,2 \mathrm{mg} / \mathrm{L}$, com diferenças nos tempos avaliados, mas não entre os tratamentos $(p<0,01)$. A concentração plasmática de TRP apresentou diferenças entre os tempos e também entre os tratamentos. $O$ GC diferiu dos demais nos momentos $48 \mathrm{~h}$ e $60 \mathrm{~h}$ e as con- 
centrações nos grupos GK e GFM foram menores que nos grupos GF e GC às $72 h(P=0,0012)$. Conclui-se que nas doses utilizadas os antiinflamatórios não esteroidais avaliados não interferem nas concentrações de TYR e PEA. Entretanto, o ketoprofeno e o flunixin meglumine foram efetivos em diminuir a concentração plasmática de TRP.

TERMOS DE INDEXAÇÃO: Aminas, antiinflamatório, laminite, eqüino.

\section{INTRODUÇÃO}

Aminas biogênicas (BA) são bases orgânicas com anéis alifáticos (putrescine, cadaverine, spermine, espermidina), aromáticos (tiramina, feniletilamina) ou heterocíclicos (histamina, triptamina), produzidas principalmente por descarboxilação microbiana de aminoácidos (Sillas Santos 1996). Essas exotoxinas são aminas encontradas no conteúdo intestinal de animais monogástricos e poligástricos (Hill et al. 1970, Bailey et al. 2003b).

Dietas ricas em carboidratos favorecem o crescimento das populações de Lactobacillus sp e Streptococcus bovis, aos quais foi associado ao aumento da concentração de aminas bioativas em eqüinos (Bailey et al. 2003a). Variações expressivas nas concentrações de triptamina (TRP), tiramina (TYR) e feniletilamina (PEA) foram verificadas no soro de eqüinos obtidos no inverno, alimentados com feno, e no verão, alimentados em pastagens ricas em fructanos(Bailey et al. 2003b).

Elliott et al. (2003) estudando os efeitos vasoativos das aminas triptamina, tiramina e feniletilamina concluíram que elas são potentes vasoconstritoras tanto em artérias como em veias, sendo a triptamina e a tiramina vasoconstritoras seletivas, ou seja, são mais potentes em veias que artérias.

Apesar de muitas pesquisas serem conduzidas continuamente, por vários grupos, em diversos países, ainda não foram elucidados todos os mecanismos envolvidos na patofisiologia da laminite. Entretanto, segundo Mungall (2001), aminas bioativas produzidas por $S$. bovis podem induzir a degradação enzimática da membrana basal lamelar. Baseado nestas informações este estudo teve como objetivo avaliar as concentrações das aminas triptamina, tiramina e feniletilamina em eqüinos com laminite induzida por sobrecarga de carboidrato e tratados com os antiinflamatórios ketoprofeno, fenilbutazona e flunixin meglumine.

\section{MATERIAL E MÉTODOS}

Foram utilizados 20 eqüinos provenientes do município de Canaã, MG, hígidos (Equus caballus caballus), sem raça definida, machos castrados, com idade entre 3 e 15 anos, peso entre 264 e $344 \mathrm{~kg}$ e escore corporal de dois a três (Speirs 1997). Antes do experimento os animais passaram por quarentena, período em que foram avaliados, pesados e numerados de 1 a 20 , sendo mantidos em piquete coletivo até sete dias antes da indução de laminite, recebendo água $a d$ libitum, feno de coast cross e ração concentrada (Nutriage-
Guabi, $15 \%$ de proteína), duas vezes ao dia, em quantidades equivalentes a $2,5 \%$ e $1 \%$ de peso corporal, respectivamente. À ração foram adicionados $50 \mathrm{~g}$ de sal mineralizado para eqüinos 7 dias antes da indução experimental os animais foram alojados em cocheiras individuais, privados da ração comercial e passaram por controle de endo e ectoparasitos, administrandose uma base conjugada de ivermectina-pomoatos de praziquantel-pirantel ${ }^{6}$ e deltametrina ${ }^{7}$ respectivamente. Após a sobrecarga de carboidrato, os animais receberam suplemento eletrolítico ${ }^{8}$ (25g/10L de água) adicionado diariamente à água de beber.

\section{Indução de laminite}

Após jejum alimentar e hídrico de $12 \mathrm{~h}$, os animais receberam amido de milho ${ }^{9}(20 \mathrm{~g} / \mathrm{kg})$, segundo modificação do modelo experimental referido por Weiss et al. (1998). Cada kilograma de amido foi dissolvido em um litro de água, sendo passado via sonda nasogástrica $(11 \mathrm{~mm})$. Imediatamente após a administração do amido os animais voltaram a receber o feno como anteriormente descrito.

\section{Grupos experimentais}

Os animais foram aleatoriamente divididos em quatro grupos experimentais $(n=5)$ e, $36 \mathrm{~h}$ após a indução da laminite, receberam, a cada 12h; Grupo 1, ketoprofeno ${ }^{10}$ (GK) 2,2mg/ kg.; Grupo 2, fenilbutazona ${ }^{11}$ (GF) 4,4mg/kg; Grupo 4 flunixin meglumine $^{12}$ (GFM) $1,1 \mathrm{mg} / \mathrm{kg}$ e Grupo 4 , solução salina ${ }^{13}$ (GC)10mL. A via de administração utilizada foi a iv, sempre na jugular esquerda, preservando-se a direita para a coleta das amostras de sangue, utilizando-se frascos a vácuo, contendo EDTA $^{14}$. As amostras foram colhidas antes da indução, seis horas após e a cada 12 horas até completar 72 horas de avaliação, totalizando oito amostras em cada repetição $(0,6$, $12,24,36,48,60$ e 72 h).

\section{Extração de aminas bioativas}

Partindo-se das amostras de $1,5 \mathrm{~mL}$ de plasma colhidas em tubos com EDTA, promoveu-se a precipitação de proteínas através da adição de $100 \mu \mathrm{l}$ de solução de ácido trifluoroacético ${ }^{15}$ a $10 \mathrm{moL}$. As amostras foram então centrifugadas ${ }^{16}$ a $12.000 \mathrm{rpm}$, por $15 \mathrm{~min}$. Uma alíquota de $0,200 \mathrm{~mL}$ do sobrenadante foi transferida para tubos criogênicos ${ }^{17}$ e submetidos a liofilização e armazenadas em freezer a $-20^{\circ} \mathrm{C}$.

O material liofilizado foi ressuspendido em $100 \mu \mathrm{L}$ de solução de anidrido trifluoroacético/acetonitrila na proporção de 1:1. Esta solução foi homogeneizada em vórtex e aquecida ao microondas ${ }^{18}$ por $3 \mathrm{~min}$ a $60 \%$ da potência total do aparelho. Os derivados acilados foram secos em linha de nitrogênio e em

\footnotetext{
${ }^{6}$ Centurion-Valleé, Brasil.

7 Butox-Intervet, Brasil.

8 Plusvital-Marcolab, Brasil.

${ }^{9}$ Milhena-Nutril, Brasil

${ }^{10}$ Ketofen-Merial, Brasil.

${ }^{11}$ Equipalazone-Marcolab, Brasil.

12 Flumegan-Marcolab, Brasil.

${ }^{13}$ Solução salina 0,9\%-Sanobiol, Brasil.

${ }^{14}$ Vacutainer-Bacton and Dickinson, Brasil.

${ }^{15}$ Acido tricloroacético PA/ACS Cristal (marca Synth).

${ }^{16}$ Centrífuga 5415 D Eppendorf.

17 Tubo criogênico c/ tampa de rosca, estéril (marca Alfa).

${ }^{18}$ Panasonic Píccolo $600 \mathrm{~W}$.
} 
seguida retomados com $50 \mu \mathrm{L}$ de acetonitrila marcada com padrão interno ${ }^{19}, 2 \mu \mathrm{L}$ desta solução foram injetados no cromatógrafo $^{20}$ para análise.

\section{Cromatografia gasosa}

As condições de operação foram $150^{\circ} \mathrm{C}, 1 \mathrm{~min}, 7^{\circ} \mathrm{C} / \mathrm{min}, 220$ ${ }^{\circ} \mathrm{C}, 20^{\circ} \mathrm{C} / \mathrm{min}, 270^{\circ} \mathrm{C}$, injetor e detector a $280^{\circ} \mathrm{C}$, Split $1 / 100$, sendo utilizada a coluna capilar ${ }^{21}$. Como gás de arraste utilizouse o hidrogênio. Foram preparados quatro padrões de cada uma das aminas analisadas, triptamina (TRP), tiramina (TYR,) e feniletilamina (PEA) (Fig.1). As correlações entre a resposta obtida e a concentração nominal das aminas e o padrão interno

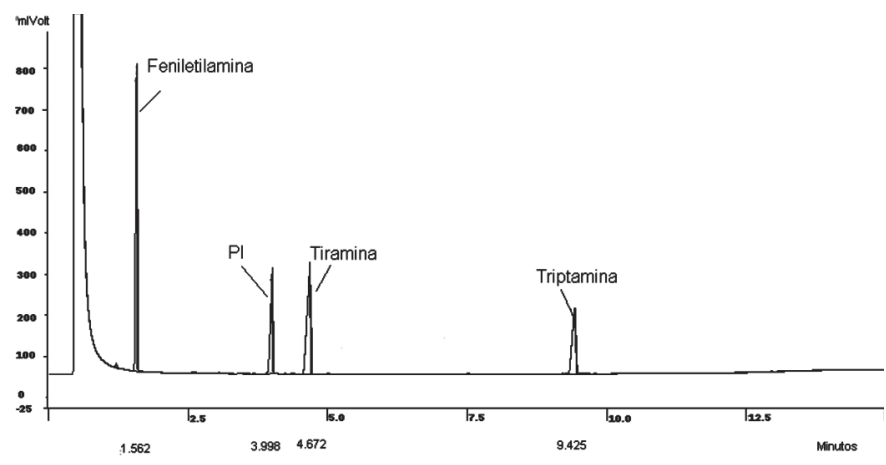

Fig.1. Cromatograma com os padrões de feniletilamina, padrão interno $(\mathrm{PI})$, de tiramina e de triptamina.

foi realizada a partir de das concentrações de PEA $(0,42 ; 0,84$; $1,25 ; 1,67 \mathrm{mg} / \mathrm{mL}) ;$ TYR $(0,248 ; 0,495 ; 0,743 ; 0,990 \mathrm{mg} / \mathrm{mL}) ;$ TRP $(0,305 ; 0,610 ; 0,915 ; 1,220 \mathrm{mg} / \mathrm{mL})$ e o padrão interno $(\mathrm{C} 18)$ a uma concentração constante de $0,74 \mathrm{mg} / \mathrm{mL}$ :

Concentração em mg/L $=\left(A_{a} / A_{p}\right) / F_{c i} \times C_{p} \times F$

$\mathrm{Aa}=$ área do pico correspondente à amostra;

$\mathrm{Ap}=$ área do pico correspondente ao padrão interno;

$\mathrm{F}_{\mathrm{ci}}=$ fator de correção interno;

$\mathrm{Cp}=$ concentração da solução padrão interno (mg/L);

$\mathrm{F}=$ fator de diluição da amostra durante o processamento.

\section{Análise estatística}

Os resultados foram avaliados quanto à normalidade, apresentando dispersão não normal e não foi possível a transformação das variáveis. Os dados foram então analisados pelo teste de Kruskal-Wallis com nível de significância de 5\%. Empates foram encontrados nos dados e as correções apropriadas foram aplicadas. As médias foram submetidas a comparações múltiplas pareadas utilizando-se o procedimento de Dunn/ Teste bilateral.

\section{RESULTADOS E DISCUSSÃO}

As concentrações plasmáticas de TYR e PEA apresentaram variação semelhante nos tempos observados, elevando-se nas primeiras $6 \mathrm{~h}(\mathrm{p}=0,0012)$ após o fornecimento de $20 \mathrm{~g} / \mathrm{kg}$ de amido aos eqüinos. As médias variaram de

\footnotetext{
${ }^{19} \mathrm{C} 18$ - Octadecano 0,74g/L.

20 Cromatógrafo a gás Varian CP-3380.

21 BP10 ( $14 \%$ cyanopropylphenyl dimethyl siloxane) $25 \mathrm{~m} \times 0.22 \mathrm{~mm}$ x 0,25um (SGE).
}

2,7 e 6,21 mg/L para a TYR e PEA, respectivamente, na hora 0, para 682,8 e 124,9mg/L no tempo 6h (Quadros 1 e 2).

A oferta de amido em quantidade elevada favorece o desenvolvimento de bactérias fermentativas e a concentração das aminas aumenta em proporção direta à população microbiana (Chin \& Koehler 1986, Bailey et al. 2003a). Bailey et al. (2003b) sugeriram que a sobrecarga por carboidratos pode favorecer a absorção de aminas pelo trato digestivo, devido ao dano causado à barreira presente na mucosa intestinal, sendo que a tiramina e a feniletilamina foram apontadas como iniciadoras da hipertensão subseqüente. Nout (1994) salientou que a tiramina em alimentos deve estar em concentração entre 100$800 \mathrm{mg} / \mathrm{kg}$, pois se torna tóxica acima de $1080 \mathrm{mg} / \mathrm{kg}$.

Seguindo-se o pico observado 6h após a administração do amido nos eqüinos deste estudo, as concentrações regrediram a 0,11 e 1,05mg/L para TYR e PEA às $24 \mathrm{~h}$, chegando a 1,04 e 0,95 $72 \mathrm{~h}$ após a administração de amido (Quadros 1 e 2). Não houve diferença entre os tratamentos, mas sim entre os tempos avaliados. Bailey et al. (2003b) observaram concentrações plasmáticas variando de $10 \mathrm{pM}$ a $100 \mathrm{nM}$ em pôneis normais e com laminite, respectivamente, valores muito menores que os

\begin{tabular}{|c|c|c|c|c|}
\hline \multicolumn{5}{|c|}{$\begin{array}{c}\text { Quadro 1. Concentrações séricas de feniletilamina em } \\
\text { eqüinos sob efeitos de sobrecarga de carboidratos e } \\
\text { tratados com ketoprofeno (GK) } 2,2 \mathrm{mg} / \mathrm{kg} \text {, fenilbutazona } \\
\text { (GF) } 4,4 \mathrm{mg} / \mathrm{kg} \text {, flunixin meglumine (GFM) } 1,1 \mathrm{mg} / \mathrm{kg} \text { e } \\
\text { solução salina (GC) } 10 \mathrm{~mL}\end{array}$} \\
\hline Hora & GK & GF & GFM & GC \\
\hline 0 & 3,72 abcA & $13,97 \mathrm{aA}$ & 2,36 aA & $13,17 \mathrm{aA}$ \\
\hline 6 & $190,10 \mathrm{aA}$ & $256,37 \mathrm{aA}$ & $182,36 \mathrm{aA}$ & $156,70 \mathrm{aA}$ \\
\hline 12 & $3,92 \mathrm{abA}$ & $6,36 \mathrm{aA}$ & $2,30 \mathrm{abA}$ & $17,33 \mathrm{aA}$ \\
\hline 24 & $3,74 \mathrm{abcA}$ & $1,14 \mathrm{bA}$ & $1,97 \mathrm{bA}$ & $2,14 \mathrm{abA}$ \\
\hline 36 & 2,37 bcA & $1,99 \mathrm{bA}$ & $9,44 \mathrm{aA}$ & $3,43 \mathrm{abA}$ \\
\hline 48 & 0,76 bcA & $1,12 \mathrm{bA}$ & $3,01 \mathrm{aA}$ & $1,97 \mathrm{abA}$ \\
\hline 60 & 0,22 cA & 3,00 abA & $0,57 \mathrm{bA}$ & 1,07 bA \\
\hline 72 & $1,37 \mathrm{bA}$ & $0,71 \mathrm{bA}$ & $1,06 \mathrm{abA}$ & $0,57 \mathrm{bA}$ \\
\hline
\end{tabular}

Médias seguidas de mesmas letras (minúsculas nas colunas e maiúsculas na linha) não diferem significativamente $(p<0,0001)$.

Quadro 2. Concentrações séricas de tiramina em eqüinos sob efeitos de sobrecarga de carboidratos e tratados com ketoprofeno (GK) $2,2 \mathrm{mg} / \mathrm{kg}$, fenilbutazona (GF) $4,4 \mathrm{mg} / \mathrm{kg}$, flunixin meglumine (GFM) $1,1 \mathrm{mg} / \mathrm{kg}$ e solução salina (GC) $10 \mathrm{~mL}$

\begin{tabular}{ccccc}
\hline Hora & GK & GF & GFM & GC \\
\hline 0 & $0,27 \mathrm{aA}$ & $8,73 a b A$ & $1,32 \mathrm{aA}$ & $0,82 \mathrm{aA}$ \\
6 & $1011,95 \mathrm{bA}$ & $1384,88 \mathrm{bA}$ & $1084,01 \mathrm{bA}$ & $574,17 \mathrm{bA}$ \\
12 & $4,87 \mathrm{abA}$ & $0,32 \mathrm{abA}$ & $0,26 \mathrm{aA}$ & $10,02 \mathrm{abA}$ \\
24 & $0,36 \mathrm{aA}$ & $0,07 \mathrm{aA}$ & $0,22 \mathrm{aA}$ & $0,25 \mathrm{aA}$ \\
36 & $1,25 \mathrm{abA}$ & $0,49 \mathrm{abA}$ & $1,30 \mathrm{aA}$ & $1,07 \mathrm{aA}$ \\
48 & $0,29 \mathrm{aA}$ & $0,16 \mathrm{aA}$ & $0,76 \mathrm{abA}$ & $0,44 \mathrm{aA}$ \\
60 & $0,86 \mathrm{abA}$ & $0,60 \mathrm{abcA}$ & $0,59 \mathrm{abA}$ & $1,62 \mathrm{abA}$ \\
72 & $3,11 \mathrm{aA}$ & $0,93 \mathrm{aA}$ & $0,55 \mathrm{abA}$ & $0,41 \mathrm{aA}$
\end{tabular}

Médias seguidas de mesmas letras (minúsculas nas colunas e maiúsculas na linha) não diferem significativamente $(p=0,0011)$. 


\begin{tabular}{|c|c|c|c|c|}
\hline Tempo & GK & GF & GFM & GC \\
\hline 0 & $0,87 a A$ & $0,82 \mathrm{abA}$ & $0,61 \mathrm{aA}$ & 1,49 acA \\
\hline 6 & $0,21 a A$ & $0,49 \mathrm{abA}$ & $0,71 \mathrm{aA}$ & $0,51 a b A$ \\
\hline 12 & 0,74 aA & $0,35 \mathrm{abA}$ & $0,44 \mathrm{aA}$ & $0,74 a b A$ \\
\hline 24 & 0,43 aA & $0,00 \mathrm{bA}$ & 0,19 aA & 0,05 bA \\
\hline 36 & $0,14 a A$ & $0,33 \mathrm{abA}$ & 0,80 aA & $0,79 a b A$ \\
\hline 48 & 0,52 aA & $0,93 \mathrm{abA}$ & 0,67 aA & $2,28 \mathrm{cB}$ \\
\hline 60 & 2,75 aA & $2,35 \mathrm{aB}$ & $3,01 \mathrm{aB}$ & 7,20 cB \\
\hline 72 & $2,21 \mathrm{aA}$ & $6,85 \mathrm{aB}$ & 2,76 aA & $6,95 \mathrm{cB}$ \\
\hline
\end{tabular}

Médias seguidas de mesmas letras (minúsculas nas colunas e maiúsculas na linha) não diferem significativamente $(p=0,0012)$.

observados nos eqüinos deste trabalho. Tal fato certamente foi devido às diferenças metodológicas e entre as populações estudadas.

As concentrações de TRP variaram de forma distinta ao longo do tempo. Mantiveram-se estáveis até $36 \mathrm{~h}$, médias de 0,$13 ; 0,33 ; 0,80$ e $0,79 \mathrm{mg} / \mathrm{L}$, respectivamente para os tratamentos com GK, GF, GFM e GC, elevando-se para 2,$21 ; 6,85 ; 2,76$ e $6,95 \mathrm{mg} / \mathrm{L}$, no momento $72 \mathrm{~h}$, quando os tratamentos GK e GFM diferiram de GF e GC $(p=0,0011)$ (Quadro 3). O GC também diferiu dos demais grupos nos momentos $48 \mathrm{~h}$ e $60 \mathrm{~h}$. A triptamina tem efeito hipertensivo em seres humanos (Shalaby 1996). Triptamina, tiramina e feniletilamina foram identificadas como causadoras de vasoconstrição in vitro em artérias e veias digitais de eqüinos (Bailey et al. 2000, 2001). Bailey et al. (2004) infundiram triptamina $(1,6 \mu \mathrm{g} / \mathrm{kg} / \mathrm{min})$ e feniletilamina $(2,13 \mathrm{~g} /$ $\mathrm{kg} / \mathrm{min}$ ) em eqüinos e observaram decréscimo na irrigação arterial digital (media \pm d.p. $29,2 \pm 8,5$ e $18,4 \pm 6,8 \%$, respectivamente), o que resultou em decréscimo na temperatura da parede dorsal do casco $\left(0,6 \pm 0,1^{\circ} \mathrm{C}\right.$ e $\left.0,5 \pm 0,1^{\circ} \mathrm{C}\right)$ para triptamina e feniletilamina, respectivamente.

\section{CONCLUSÃO}

Os resultados obtidos sugerem que a administração dos antiinflamatórios ketoprofeno e flunixin meglumine minimiza os efeitos decorrentes da presença de triptamina na circulação sanguínea, oriundas de distúrbios entéricos fermentativos em eqüinos, entretanto, não altera as concentrações plasmáticas de tiramina e feniletilamina.

Agradecimentos.- Ao Conselho Nacional de Desenvolvimento Científico e Tecnológico (CNPq) pela bolsa de Pós-Doutorado Júnior (PDJ) e o suporte financeiro (Proc.154922/2006-4).

\section{REFERÊNCIAS}

Bailey S.R., Rycroft A. \& Marr C.M. 2000. Identification and quantification of amines in equine caecal liquor. Proc. 39th Brit. Equine Vet. Assoc. Congr., p.206-207.

Bailey S.R., Rycroft A. \& Marr C.M. 2001. Effect of carbohydrate overload on production of vasoactive amines in equine caecal contents in vitro. Proc. 40th Brit. Equine Vet. Assoc. Congr., p.213.

Bailey S.R., Baillon M.L., Rycroft A.N., Harris P.A., Elliott J. 2003a. Identification of equine cecal bacteria producing amines in an in vitro model of carbohydrate overload. Appl. Environ. Microbiol. 69(4):20872093,

Bailey S.R., Katz L.M., Berhane Y., Samuels T., De Brauvere N., Marr C.M. \& Elliott J. 2003b Seasonal changes in plasma concentrations of cecum-derived amines in clinically normal ponies predisposed to laminitis. Am. J. Vet. Res. 64:1132-1138.

Bailey S.R., Menzies-Gow N.J. \& Marr C.M. 2004. The effects of vasoactive amines found in the equine hindgut on digital blood flow in the normal horse. Equine Vet. J. 36(3):267-272.

Chin E.D. \& Koehler P.E. 1986. Effect of salt concentration and incubation temperature on formation of histamine, phenethylamine, tryptamine, and tyramine during miso fermentation. J. Food Prot. 49:423-427.

Elliot J., Berhane Y. \& Bailey S.R. 2003. Effects or monoamines formed in the cecum of horses on equine digital blood vessels and platelets. Am. J. Vet. Res. 64:1124-1131.

Hill I.R., Kenworthy R., Porter P. 1970. Studies of the effect of dietary lactobacilli on intestinal and urinary amines in pigs in relation to weaning and post-weaning diarrhea. Res. Vet. Sci. 11:320-326.

Mungall B. A., Kyaw-Tanner M. \& Pollitt C.C. 2001. In vitro evidence for a bacterial pathogenesis of equine laminitis. Vet. Microbiol. 79:209223.

Nout M.J.R. 1994. Fermented foods and food safety. Food Res. Int. 27:291-298.

Shalaby A.R. 1996. Significance of biogenic amines to food safety and human health. Food. Res. Internat. 29:675-690.

Sillas Santos M.H.1996. Biogenic amines: their importance in foods. Food Res. Int. 29:213-231.

Speirs V.C. 1997. The alimentary tract, p.261-298. In: Speirs V.C. (ed.), Clinical Examination of Horses. W.B. Saunders, Philadelphia.

Weiss D.J., Evanson A.O., McClenahan D., Fagliari J.J., Dunnwiddie C.T. \& Wells R.E. 1998. Effect of a competitive inhibitor of platelet aggregation on experimentally induced laminitis in ponies. Am. J. Vet. Res. 59:814-817. 